\title{
Optical imaging of the spatiotemporal dynamics of cerebral blood flow and oxidative metabolism in the rat barrel cortex
}

\author{
B. Weber, ${ }^{1,2}$ C. Burger, ${ }^{1}$ M. T. Wyss,${ }^{1}$ G. K. von Schulthess, ${ }^{1}$ F. Scheffold ${ }^{3}$ and A. Buck ${ }^{1}$ \\ ${ }^{1}$ Division of Nuclear Medicine, University Hospital Zurich, Rämistrasse 100, 8091 Zurich, Switzerland \\ ${ }^{2}$ Max Planck Institute for Biological Cybernetics, Spemannstraße 38, 72076 Tübingen, Germany \\ ${ }^{3}$ Department of Physics, Soft Condensed Matter Group, University of Fribourg-Perolles, Chemin du Musée, 1700 Fribourg, \\ Switzerland
}

Keywords: autofluorescence, BOLD fMRI, laser speckle

\begin{abstract}
Oxidative metabolism and cerebral blood flow (CBF) are two of the most important measures in neuroimaging. However, results from concurrent imaging of the two with high spatial and temporal resolution have never been published. We used flavoprotein autofluorescence (AF) and laser speckle imaging (LSI) in the anaesthetized rat to map oxidative metabolism and CBF in response to single vibrissa stimulation. Autofluorescence responses reflecting oxidative metabolism demonstrated a fast increase with a delay of $0.1 \mathrm{~s}$. The sign-reversed speckle contrast reflecting CBF started to rise with a delay of $0.6 \mathrm{~s}$ and reached its maximum $1.4 \mathrm{~s}$ after the stimulation offset. The fractional signal changes were $2.0 \%$ in AF and $9.7 \%$ in LSI. Pixelwise modelling revealed that CBF maps spread over an area up to 2.5 -times larger than metabolic maps. The results provide evidence that the increase in cerebral oxidative metabolism in response to sensory stimulation is considerably faster and more localized than the CBF response. This suggests that future developments in functional imaging concentrating on the metabolic response promise an increased spatial resolution.
\end{abstract}

\section{Introduction}

Functional brain imaging is an exceptional success story in the neurosciences. This is partly because some methods such as functional magnetic resonance imaging (fMRI) and positron emission tomography (PET) allow the noninvasive imaging of the human brain. However, the fundamentals of some of the measured signals are still poorly understood. To study these fundamentals invasive imaging in the animal brain is still mandatory, because of the superior temporal and spatial resolution of some of the available techniques, and because imaging can be combined with microelectrode recordings of neuroelectric activity.

Optical imaging is one of the most powerful tools to obtain highresolution functional maps (Grinvald et al., 1986; Bonhoeffer \& Grinvald, 1996). Many of the optical imaging techniques including some intrinsic optical measures are based on the haemodynamic response to neural activation. However, a method to map regional cerebral blood flow (CBF) itself with a high spatial and temporal resolution, namely laser speckle imaging (LSI), has been introduced only recently (Dunn et al., 2001, 2003). The underlying principle of LSI is equivalent to the one exploited in laser Doppler flowmetry (LDF), a method that is accepted to be the gold standard to serially measure relative changes of $\mathrm{CBF}$, however, only from one point in space at a time. Both methods are described in detail elsewhere (Briers, 2001). Briefly, laser light scattered from tissue provides sensitive information about the movement inside a surface layer. A

Correspondence: Dr Bruno Weber, ${ }^{2}$ Max Planck Institute for Biological Cybernetics, as above.

E-mail: bruno.weber@tuebingen.mpg.de

Received 8 July 2004, revised 9 September 2004, accepted 13 September 2004 moving object inside the tissue, such as a red blood cell, leads to a Doppler shift of the frequency of scattered light that is directly proportional to the blood cell velocity. Rather than analysing frequency shifts LSI looks at temporal fluctuations of the scattered light in order to map blood flow. From a fundamental point of view both techniques are equivalent. The frequency and time domain in light scattering are connected by a simple Fourier transformation and thus completely interchangeable (Berne \& Pecora, 1976; Briers, 2001; Dunn et al., 2001). In other words if the spectrum of frequency shifts is known the distribution of temporal fluctuations can be calculated and vice versa.

In LSI a CCD camera records the intensity pattern of light reflected from the tissue. In the absence of movement (or for very short exposure time) there is no detectable fluctuation and the typical granular interference pattern, called speckle, has the same properties in the entire field of view. The pattern represents a distinct fingerprint of the spatial configuration of all scattering objects in the medium, such as tissue, blood cells, etc. Changes in the position of some objects lead to temporal intensity fluctuations with a time constant given by the local velocity. Instead of analysing the temporal fluctuations it is more convenient to measure a time average simply by choosing a certain camera exposure time. The speckle contrast (defined as $\mathrm{K}=\mathrm{s} /<\mathrm{I}>$, where $\mathrm{s}$ is the standard deviation and $<\mathrm{I}>$ is the mean intensity in an area) is then a measure of the average local velocity - a high contrast corresponds to no movement whereas a low contrast is found in areas of high movement. This very promising new method is relatively easy to implement and has been used to image the CBF response to spreading depression and single vibrissa stimulation (Dunn et al., 2001, 2003) as well as to peripheral electrical stimulation (Durduran et al., 2004) in the rat cortex. 
It is well established that the mitochondrial redox state and with it the oxidative metabolism can be monitored in vivo using autofluorescence (AF) imaging (Lothman et al., 1975; Mayevsky \& Chance, 1975; Lewis \& Schuette, 1976; Barlow et al., 1979; Mayevsky \& Chance, 1983; Haselgrove et al., 1990). Both NADH and flavoproteins change their fluorescence property from the oxidized to the deoxidized form. In their seminal report Shibuki and collegues exploited the flavoprotein autofluorescence states to dynamically image the rat cortex both in vitro and in vivo with excellent spatial and temporal resolution (Shibuki et al., 2003). Reinert et al. (2004) used the same method in the mouse cerebellar cortex and further evaluated its relationship to the mitochondrial metabolism. In a recent paper, Murakami et al. (2004) employed flavoprotein AF imaging to study short-term plasticity in the somatosensory cortex of the rat. Flavoprotein $\mathrm{AF}$ imaging was shown to have a superb signal-to-noise ratio (SNR), allowing the analysis of single trials. To excite green flavoprotein AF (500-550 nm), illumination with a wavelength of $450-490 \mathrm{~nm}$ is appropriate. It is important to note that at the wavelength of $500-550 \mathrm{~nm}$ the absorption by haemoglobin is high and thus the $\mathrm{AF}$ is confounded by the haemodynamic response (Shibuki et al., 2003). Therefore only the initial phase of the signal can be used to exclusively extract information on the metabolic state. Using the rat barrel cortex as a well-established model system, we have combined laser speckle and AF imaging for the first time. This combination allows the extraction of information on $\mathrm{CBF}$ and oxidative metabolism in exactly the same field of view with a high spatial and temporal resolution. The purpose of this study was to contribute to a better understanding of the basic relationship between $\mathrm{CBF}$ and oxidative metabolism, two important parameters in functional neuroimaging that greatly influence extensively used measures such as the BOLD fMRI signal.

\section{Materials and methods}

\section{Animal preparation}

Ten male Sprague-Dawley rats (250-350 g) were used (excluding preliminary experiments). Surgery was performed under isoflurane anaesthesia whereas during the actual experiment the rat was anaesthetized using $\alpha$-chloralose (Sigma C-9821, $44 \mathrm{mg} / \mathrm{kg}$ s.c.) and $0.3-0.5 \%$ isoflurane. $\alpha$-Chloralose was chosen because of its relatively small effect on cerebrovascular physiology (Bonvento et al., 1994). Isoflurane was used during the surgical procedure for its analgesic effect and then during the experiment to fine-tune the depth of anaesthesia. Catheters were placed in the right femoral artery and vein for the continuous monitoring of the arterial blood pressure (90-110 $\mathrm{mmHg})$, for the i.v. administration of mannitol $(1 \mathrm{~g} / \mathrm{kg}$, to prevent brain oedema) and of a lethal dose of pentobarbital at the end of the experiment. The animals were tracheotomized and ventilated mechanically to maintain physiological arterial levels of $\mathrm{pH}(7.35$ 7.45), $\mathrm{pCO}_{2}$ (34-38 mmHg) and $\mathrm{pO}_{2}(>100 \mathrm{mmHg})$. The body temperature was held constant at $37{ }^{\circ} \mathrm{C}$ using a regulated heating pad. The animals were placed in a stereotaxic holder (David Kopf Instruments, Tujunga, CA, USA) and the left somatosensory cortex was exposed and covered with agar ( $2 \%$ in saline). All experimental procedures were approved by the local veterinary authorities.

\section{Stimulation and experimental protocol}

Single vibrissae (right C2, D2) were deflected using a canula attached to a piezoelectric ceramic wafer (Piezo Systems Inc., Cambridge, MA, USA). The stimulator was fixed to an independent holder (independent from the operating table) to avoid even subtle vibrations that potentially disturb the imaging (particularly the laser speckle imaging). The stimulated vibrissae were clipped to a length of $10 \mathrm{~mm}$ and the remaining vibrissae were cut at the skin surface. The tip of the canula was placed $5 \mathrm{~mm}$ from the base of the vibrissa. A computer triggered pulse $(50 \mathrm{~ms})$ sent to the wafer produced a $1 \mathrm{~mm}$ rostral-tocaudal deflection of the vibrissae. The stimulation lasted $2 \mathrm{~s}$ with a frequency of $2 \mathrm{~Hz}$. Before stimulation, a baseline phase of $1 \mathrm{~s}$ was acquired. A total of 20 trials were recorded before the stimulated vibrissa was changed. Speckle contrast imaging and AF imaging were alternated from trial to trial, giving a total of 10 trials per modality. A 45-s interval followed each trial to allow the signals to return to baseline. During constant application of excitation light the AF signal decreases, a common process of all fluorescence imaging methods, which is referred to as bleaching. Thus such a long intertrial interval has been chosen to allow flavoproteins to recover from bleaching.

\section{Optical imaging}

Cortical images were acquired using a 12-bit CCD camera (Pixelfly VGA, PCO Imaging, Kelheim, Germany) attached to a motorized epifluorescence stereomicroscope (Leica MZ16 FA, Leica Microsystems, Heerbrugg, Switzerland) focused $0.5 \mathrm{~mm}$ below the cortical surface. For AF and LSI $240 \times 320$ pixels (using $2 \times 2$ binning) were acquired. For flavoprotein AF imaging the cortex was illuminated with blue light (excitation filter band-width 450-490 nm) and green images (barrier filter band width 500-550 nm) were collected. The excitation light was switched on $5 \mathrm{~s}$ before and was stopped simultaneously with the end of each image acquisition sequence. In $7.5 \mathrm{~s} 60$ frames were acquired with a frequency of $8 \mathrm{~Hz}$ and an exposure time of $125 \mathrm{~ms}$. The excitation light and data recording was restricted to this interval to minimize bleaching effects. For the LSI, the cortex was illuminated with a $785 \mathrm{~nm}$ laser feeding a single-mode fibre attached to a collimating lens (Schäfter und Kirchhoff, Hamburg, Germany). In $15 \mathrm{~s}$ 1500 frames (exposure time $10 \mathrm{~ms}$ ) were acquired. The speckle contrast (defined as $\mathrm{K}=\mathrm{s} /<\mathrm{I}>$, where $\mathrm{s}$ is the standard deviation and $<\mathrm{I}>$ is the mean intensity in an area) was computed using a bin size of $5 \times 5$ pixels. A total of 12 consecutive contrast images were then averaged to increase the SNR and to adjust the time scale to the AF signal. All LSI data were sign-reversed to reflect blood flow and to allow a more straightforward comparison with the AF data. Data acquisition, stimulation triggering and all the manipulations of the microscope (shutter and filterwheel positioning) were automatically controlled by a linux workstation using Matlab (The MathWorks, Natick, MA, USA).

\section{Image analysis and statistics}

The data matrices (AF and speckle contrast images) were reduced to a size of $120 \times 160$ pixels, spatially filtered using a Gaussian convolution (FWHM $0.12 \mathrm{~mm}$ ) and the 10 trials were averaged before they were entered in the pixelwise analysis. The pixelwise modelling was performed using our software PMOD (http://www.pmod.com). A gamma function of the form $f(t)=\mathrm{A} t^{\mathrm{B}} \times \mathrm{e}^{-t \mathrm{C}}$ (where $t$ denotes the time and $\mathrm{A}, \mathrm{B}$ and $\mathrm{C}$ the fitted parameters) was fitted to the baseline corrected data. From the resulting $\mathrm{A}, \mathrm{B}$ and $\mathrm{C}$ several additional parameters were analytically calculated and used to produce parametric maps: maximum, maximum slope and time to maximum. The time point of the start of the signal increase was defined as the time point when the fitted gamma curve crossed the line defined by mean plus one standard deviation of the baseline points $\left(t_{\text {thres }}\right)$. The spatial extent 
of the activations were quantified by measuring the area of pixels activated $>50 \%$ or $>75 \%$ of the pixel with maximum signal change. Pair-wise comparisons between AF and LSI parameters were statistically done using the Student's $t$-test for paired samples. To identify potential independent components of the LSI response, the signal was decomposed using a principal component analysis (Vanzetta et al., 2004). For an estimate of the SNR the maximum of the gamma curve was normalized to the standard deviation of the baseline points. The centre of activation was defined as the centre of mass of the 25 pixels with the highest activation.

\section{Results}

\section{Flavoprotein $A F$}

An example of the AF response is shown on the left side in Fig. 1. A very circumscribed signal increase is followed by a more distributed long-lasting decrease. The AF signal increases almost immediately following the onset of the vibrissa deflections. The mean fitted gamma curve of all experiments in the area of a signal change $>75 \%$ of peak activation is shown in Fig. 2A. It is important to note that it is the initial segment $(<2 \mathrm{~s}$ following stimulus onset) that represents the metabolic response (i.e. not confounded by the haemodynamic response; Shibuki et al., 2003) and that the fit to the AF signal was therefore limited to this phase. On average, the area with highest response ( $>50$ and $>75 \%$ of the peak activation in the whole field of view) showed a $1.58(>50 \%)$ and $1.97 \%(>75 \%)$ increase from baseline (see Table 1). The mean time to the maximum of the gamma curve, and mean $t_{\text {thres }}$ are shown in Table 1 . The spatial extent of the response at the time-point of highest activation as determined by the maximum of the fitted gamma curve is demonstrated in Fig. 3. This map is almost identical to the map obtained from the maximum slope of the fitted gamma curve (data not shown). The example parametric maps shown in Fig. 3 demonstrate that the $\mathrm{C} 2$ and D2 stimulation is well discriminated. The mean distance between the centres of activations of barrel C2 and D2 was $503 \pm 212 \mu \mathrm{m}$ (mean \pm SD).

\section{Speckle contrast imaging (LSI)}

An example of the laser speckle contrast response is shown on the right in Fig. 1. As expected the sensory stimulation markedly reduced the speckle contrast, indicating an increase in blood flow. The sign- reversed time-activity curve starts to rise with a noticeable delay and peaks only approximately $1.5 \mathrm{~s}$ after the stimulation is stopped (Fig. 2B). The area with highest blood flow increase ( $>50$ and $>75 \%$ of the peak activation) showed on average an increase of 7.76 and $9.66 \%$ from baseline (see Table 1). The spatial extent at the timepoint of the largest response is shown on the right of Fig. 3. Although the sensory stimulation produced a large area of increased blood flow, the peaks of the activations following $\mathrm{C} 2$ and $\mathrm{D} 2$ deflection can still be discriminated. The mean distance between the centres of activations of barrel C2 and D2 was $537 \pm 274 \mu \mathrm{m}$ (mean $\pm \mathrm{SD}$ ). The results of the principal component analysis are shown in Fig. 4. Only the second principal component shows a reliable and meaningful change over time that strongly resembles the fitted response shown in Fig. 2A. Furthermore, the example map derived from the second principal component matches the map from the gamma curve fit shown in Fig. 3.

\section{Comparison between flavoprotein AF and speckle contrast imaging}

\section{Response magnitude and SNR}

The percentage increase from baseline is significantly higher in the LSI than in the AF signal (see Table 1). However, the SNRs are superb in both methods and render single trial analysis feasible (see Fig. 2C and $\mathrm{D}$ and Table 1). There was no statistically significant difference between AF and LSI with respect to the SNR.

\section{Temporal aspects}

The temporal characteristics of the metabolic and haemodynamic responses are illustrated in Fig, 2 and summarized in Table 1. The onset as well as the maximum of the fitted gamma curve is significantly later in the LSI as compared to the AF response. The peak of the AF signal was reached already $1.5 \mathrm{~s}$ after the stimulation onset, which is $0.5 \mathrm{~s}$ before the end of the stimulation.

\section{Spatial aspects}

The spatial extent of the signal response to the single vibrissa stimulation was significantly larger in LSI as compared with AF, as demonstrated in Figs 1, 2B and 3. The time-course of the size of the activated area shown in Fig. 2B demonstrates that the area at peak activation is about 2.5-times smaller in AF compared with LSI. The

TABLE 1. AF and LSI responses of pixels showing $>50 \%$ and $>75 \%$ of maximum relative response

\begin{tabular}{|c|c|c|c|c|}
\hline & \multirow[b]{2}{*}{$\mathrm{AF}$} & \multirow[b]{2}{*}{ LSI } & \multicolumn{2}{|c|}{ AF vs. LSI } \\
\hline & & & $T$-value & $P$-value \\
\hline \multicolumn{5}{|c|}{ Mean relative responses of pixels showing $>50 \%$ and $>75 \%$ of maximum relative response (\%) } \\
\hline$>50 \%$ & $1.58 \pm 0.71$ & $7.76 \pm 2.60$ & 15.39 & $<0.001$ \\
\hline$>75 \%$ & $1.97 \pm 0.78$ & $9.66 \pm 3.62$ & 13.19 & $<0.001$ \\
\hline \multicolumn{5}{|c|}{ Time to maximum of fitted gamma curve $(\mathrm{s})$} \\
\hline$>50 \%$ & $1.38 \pm 0.31$ & $3.42 \pm 0.56$ & 14.70 & $<0.001$ \\
\hline$>75 \%$ & $1.49 \pm 0.45$ & $3.41 \pm 0.56$ & 13.76 & $<0.001$ \\
\hline \multicolumn{5}{|c|}{ Time-point where fitted gamma curve exceeds 1 SD of baseline (s) } \\
\hline$>50 \%$ & $0.16 \pm 0.15$ & $0.66 \pm 0.26$ & 9.80 & $<0.001$ \\
\hline$>75 \%$ & $0.14 \pm 0.14$ & $0.66 \pm 0.33$ & 7.50 & $<0.001$ \\
\hline \multicolumn{5}{|c|}{ Signal-to-noise ratio defined as maximum of fitted gamma curve expressed as SD of baseline } \\
\hline$>50 \%$ & $16.22 \pm 8.16$ & $12.92 \pm 4.83$ & 1.92 & $>0.05$ \\
\hline$>75 \%$ & $19.12 \pm 9.92$ & $16.06 \pm 6.31$ & 1.42 & $>0.05$ \\
\hline
\end{tabular}

Data are presented as mean $\pm \mathrm{SD}$. AF and LSI were compared using a T-test for paired samples. Time-point 0 represents the onset of the stimulation. 


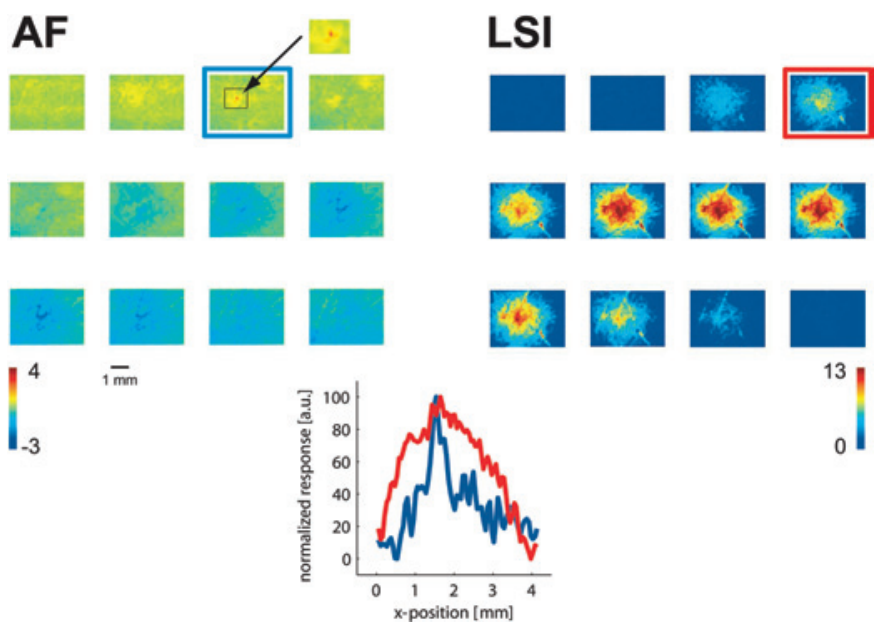

FIG. 1. Example of time evolution of AF and LSI. Each pseudocolour image shows the percentage increase from baseline. One frame represents $0.5 \mathrm{~s}$ (mean of four images). The first frame starts at the same time as the stimulation. Already the visual analysis reveals that the activated area is considerably smaller in AF (red area in frame 3, see arrow and enlarged view) compared with LSI. The bottom graph shows the activation profile (AF blue, LSI red) along the $x$-axis through the most activated pixel of the marked frame $(1.5 \mathrm{~s}$ after stimulation onset in $\mathrm{AF}$ and $2 \mathrm{~s}$ in LSI).
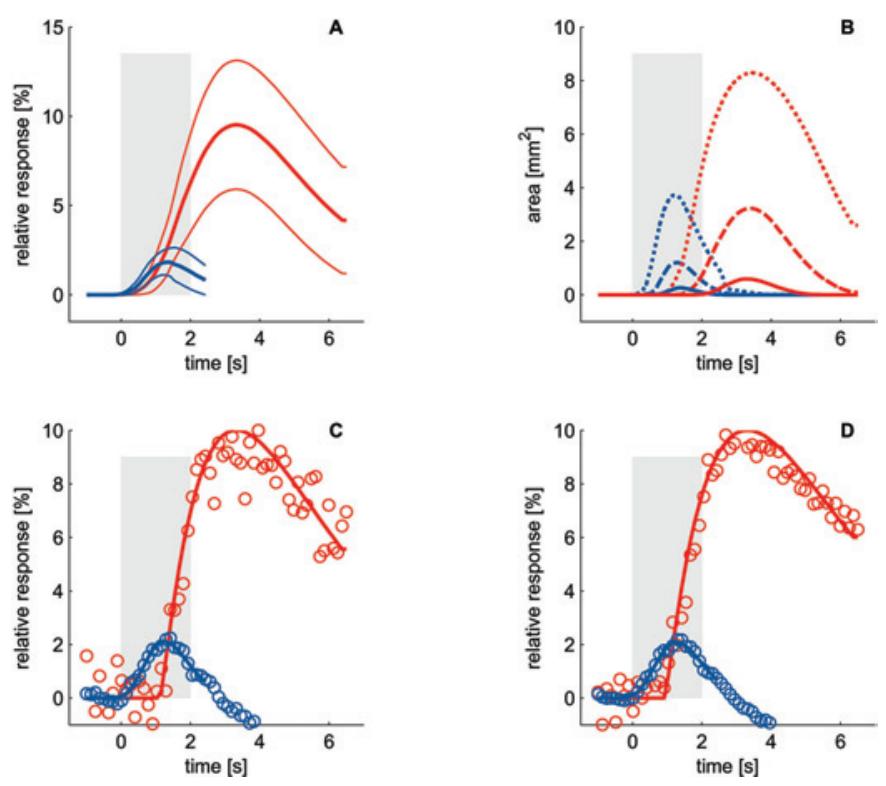

FIG. 2. Time-course of AF and LSI. (A) The mean fitted gamma curves (thick lines) \pm 1 standard deviation (thin lines) of the AF (blue) and LSI (red). The data represent the mean of all experiments in an area showing at least $50 \%$ of the peak activation of $\mathrm{C} 2$ and D2 stimulations (in 10 animals, yielding $n=20$ ). The shaded area shows the stimulation phase. The fit of the gamma curve in the AF signal is limited to the initial phase. (B) Mean time course of the size of the activated area for AF (blue) and LSI (red). Shown are the areas with $>25 \%$ (solid), $>50 \%$ (dashed) and $>75 \%$ (dotted) of the peak response. (C) Normalized time activity curves from a single trial (2 s stimulation of vibrissa $\mathrm{C} 2$, pixels averaged that showed $>50 \%$ of peak activation). The original data are represented by the markers (AF blue squares; LSI red circles). The solid lines are the fitted gamma curves. (D) Average time activity curves from all 20 trials of the experiment shown in C. Note the excellent agreement between the single trial and the average data.

lower panel of Fig. 1 shows an example where the spatial profile of signal increase is more circumscribed in AF even at early time points. However, as demonstrated in Fig. 2B there are initial time points

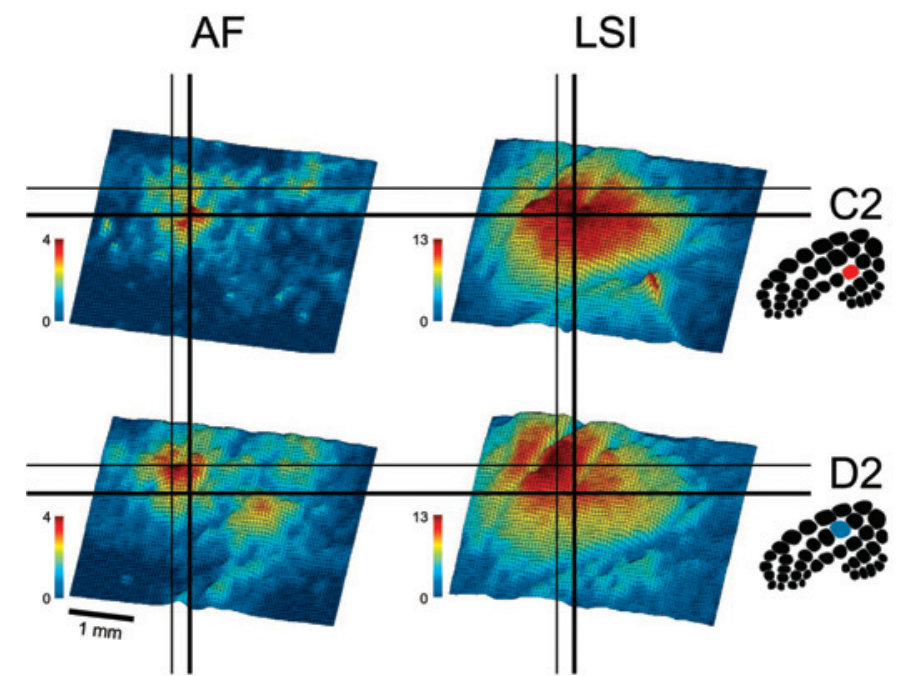

FIG. 3. Parametric maps. Example of parametric maps obtained from the maximum of the fitted gamma curve at each pixel of the AF (left) and LSI (right) signal (the data originated from 10 trials per modality). C2 stimulation are shown in the upper two maps, the D2 stimulation in the lower maps. The colour code represents the percentage increase from baseline. The horizontal and vertical lines facilitate the comparison of the peak activations across subpanels. The illustrative drawings of the barrels on the right side are not scaled correspondingly.
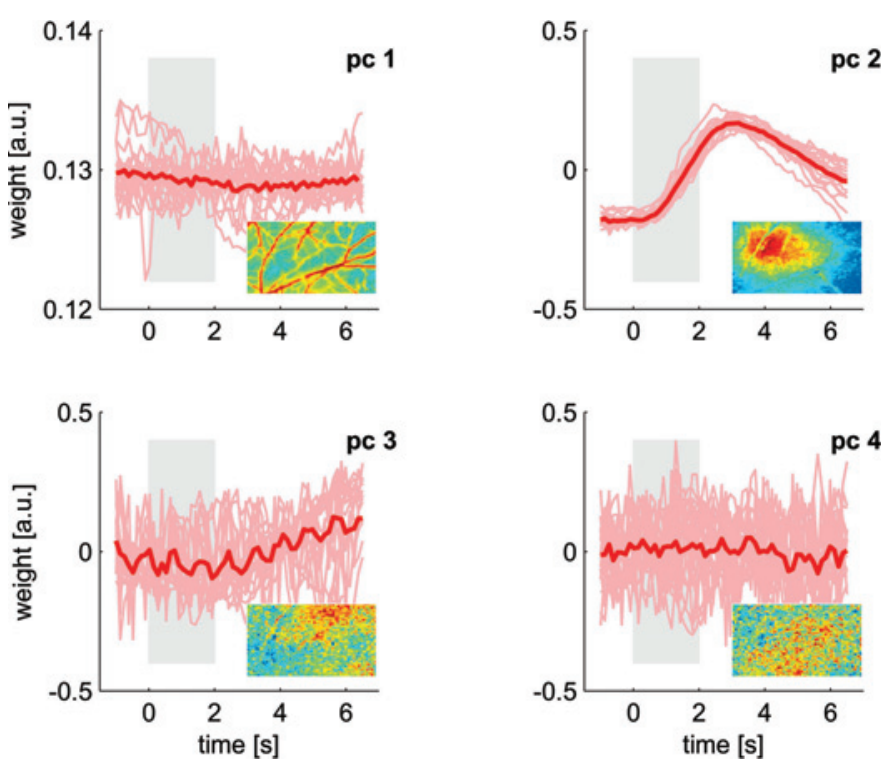

FIG. 4. Principal component analysis of LSI signal. Mean time course of the weights (eigenvectors) of the first four principal components (pc 1-4) obtained from all experiments. Insets show a representative example map obtained from the corresponding principal component. The dark red line represents the mean, the light red lines each individual trace $(n=20)$. Note that only the second component shows a reproducible change over time almost identical to the fitted response shown in Fig. 2A. The first component represents the global signal, showing very little variation over time (note different axis scale).

where the area of an increased LSI signal is smaller than the area of later AF signal increase. Nevertheless, the statistical quality of the activation maps at these early time-points is relatively poor. The spatial match between peak activations of AF and LSI maps was generally good. The distance between the centres of mass of $\mathrm{AF}$ and LSI maps were $319 \pm 214 \mu \mathrm{m}$ (mean $\pm \mathrm{SD}$ ) for $\mathrm{C} 2$ and $378 \pm 314 \mu \mathrm{m}$ for D2. This difference corresponds to somewhat more than half the known barrel diameter (Welker, 1971). 


\section{Discussion}

Both $\mathrm{CBF}$ and metabolism are considered to be two of the most important measures in functional neuroimaging. Despite this, this study is the first to present maps of high spatial and temporal resolution of these two measures acquired in the same field of view. Optical methods have been used extensively and with great success to assess neural activity with columnar resolution (Grinvald et al., 1986). Many of the intrinsic optical methods are dependent on the haemodynamic response. However, direct mapping of true CBF with high spatial resolution was only possible with autoradiographic techniques, e.g. using the $\left[{ }^{14} \mathrm{C}\right]$-iodoantipyrine method (Sakurada et al., 1978; Ohno et al., 1979) and using fMRI (Duong et al., 2001) until Dunn and colleagues had introduced LSI of neural tissue in vivo (Dunn et al., 2001). As an alternative, scanning laser Doppler approaches should be mentioned, however, their spatial and temporal resolution is still limited (Lauritzen \& Fabricius, 1995; Ances et al., 1999). A number of approaches have been proposed and successfully applied to measure oxygen consumption. Using optical methods this can be accomplished measuring the relative oxygenation of haemoglobin. Similarly, the fMRI BOLD signal can be calibrated to yield an estimation of oxygen consumption (Davis et al., 1998).

\section{Spatial pattern of changes in metabolism and CBF}

The high spatial resolution of AF has been shown previously (Shibuki et al., 2003; Reinert et al., 2004). Using the rat whisker-to-barrel system, the present study is the first to directly demonstrate that columnar sensory units can be separated easily with the flavoprotein AF method under physiological stimulation conditions. Furthermore, we demonstrate that the CBF response spreads over a larger area than the metabolic response. The area with $>75 \%$ of the maximum response was about 2.5-times as large for CBF compared to oxidative metabolism. At this threshold the mean diameter of the activated AF signal was $579 \mu \mathrm{m}$, which is only slightly larger than the published barrel size of 400-500 $\mu \mathrm{m}$ (Welker, 1971). The respective diameter measured with LSI is $868 \mu \mathrm{m}$. In this regard it should be mentioned that the activated area in the LSI map is considerably smaller at the very beginning of the signal increase (Fig. 2B). One could therefore argue that in order to achieve higher spatial resolution only this initial phase should be evaluated. However, at these early time points the statistical quality of the maps representing the signal increase is relatively poor. It remains open whether further developments in methodology (e.g. CCD with higher dynamic range or improved data analysis) could improve these early maps. Although the overlap in the activation maps of the stimulated barrels C2 and D2 is considerably larger in LSI as compared to AF, both methods yielded distinct centres of activations. The distances between these centres correspond well with the published barrel interdistance.

The activation of metabolism can be expected to better localize the site of increased neuronal activity (synaptic activity and spiking activity; Moore \& Nelson, 1998; Zhu \& Connors, 1999). In contrast $\mathrm{CBF}$ is only regulated indirectly via dilation of the vessels. It is easy to conceive that even a very localized increase in vessel size can affect CBF up- and downstream from the site of activation. This consideration suggests that cerebral blood volume (CBV) might be a superior parameter for functional imaging, which was indeed suggested by recent studies. Sheth et al. (2004b) analysed CBV changes in response to single vibrissa stimulations. They claim that with the use of a new method of data analysis CBV changes can achieve columnar specificity and that large vessel contribution becomes negligible. Based on these results we have also applied the proposed analysis of variance (ANOVA) approach but failed to improve the spatial resolution of the LSI or AF maps (data not shown). This could be due to the fact that the number of trials (10 per modality as opposed to 64 in Sheth et al., 2004b) was too small in our experiments. Columnar resolution of CBV maps was also demonstrated by employing a principal component analysis to the CBV response and extracting a component independent from the vascular response (Vanzetta et al., 2004). Inspired by that study we applied a principal component analysis to our CBF data. However, we were unable to decompose more than one functional component from the LSI signal, indicating that no component with a superior spatial resolution was present. Whether or not BOLD fMRI, which relies on an interaction between $\mathrm{CBF}, \mathrm{CBV}$ and oxygen consumption, is able to resolve single cortical columns is a question of great importance but remains elusive (for a review see Logothetis \& Wandell, 2004). Using polarographic oxygen electrodes and thus independent from the optical or fMRI approach, Thompson and collegues have confirmed the seminal studies applying intrinsic optical imaging (Frostig et al., 1990; Malonek \& Grinvald, 1996) that the early deoxygenation (initial dip) indeed reaches columnar resolution, whereas the late oxygenation spreads over several columns (Thompson et al., 2003). A similar result was reported in the rat barrel cortex using simultaneous laser speckle and intrinsic optical imaging (Dunn et al., 2003). The area with increased CBF following single vibrissa deflections was demonstrated to be considerably larger than the one obtained from haemoglobin oxygenation changes. The present study also supports the existence of an initial dip following stimulation. The increase of oxidative metabolism without concomitant rise in $\mathrm{CBF}$ should lead to a transient increase of deoxyhemoglobin.

\section{Temporal characteristics of changes in metabolism and CBF}

The immediate rise of oxidative metabolism following stimulation is in line with previous studies (Shibuki et al., 2003; Reinert et al., 2004). The delay in CBF response is in line with studies applying laser Doppler flowmetry in comparable experimental settings (Gerrits et al., 1998; Jones et al., 2002; Martindale et al., 2003; Sheth et al., 2004a).

\section{Methodology remarks}

It is somewhat surprising that the peak of the AF signal is reached before the end of the stimulation and that the AF signal might drop below baseline. An explanation is suggested by the observation that the peak of the AF signal coincides with the onset of the CBF increase. We therefore suspect that after this time-point the increased concentration of haemoglobin resulting from the $\mathrm{CBV}$ increase attenuates the AF signal. The actual peak of the metabolic response may therefore be somewhat later than the peak of the AF signal. This hypothesis is strongly supported by the finding of Shibuki et al. (2003) that the decrease of the AF signal following the start of the vascular response is less pronounced following the application of $\mathrm{N}^{\mathrm{G}}$ nitro-L-arginine, a nitric oxide synthase inhibitor. Another point in favour of the above hypothesis is the fact that no negative phase was reported in cerebral slice preparations (Shibuki et al., 2003), where no blood is present. By contrast Reinert et al. (2004) argue that the decrease of the AF signal below baseline might reflect the true timecourse of metabolism. In this context it is important to note that they performed measurements in the mouse cerebellar cortex, which is characterized by a high rate of spontaneous firing already at baseline. 
It is conceivable that the spontaneous firing rate might actually drop below baseline following stimulation, leading to a negative relative AF signal. Nevertheless, for functional imaging the existence of a time-window, during which the recorded signal has a well-defined meaning, is sufficient. For AF this is the case before the onset of the vascular response.

One might raise the concern that the spatial characteristics of the AF signal could be influenced by the inhomogeneous distribution of the flavoproteins across the barrel field, as indicated by cytochrome oxidase stainings. However, this seems unlikely because of the relatively small gap between the barrels, which is probably below the resolution of the used methods. This is supported by the result that no individual barrels were visible during baseline. Furthermore, the results represent activations as relative changes that can be expected to be minimally affected by an inhomogeneous distribution of flavoproteins.

The spatial difference between the centre of activation of the AF and LSI maps is slightly larger than half a barrel diameter (Welker, 1971). At this time it is not clear whether this reflects a methodological inaccuracy or a true physiological difference.

Another point to consider is that the neural activation during single vibrissa stimulation is not strictly limited to one barrel, as corticocortical connections and thalamic neurons projecting to the barrel cortex typically have multivibrissa receptive fields (Armstrong-James \& Callahan, 1991; Moore \& Nelson, 1998). It is therefore very possible that some of the signal recorded outside the main barrel represents true neural activity. Nevertheless, this possibility does not affect one of the major results of this study, namely the finding that the spatial extent of the CBF response is considerably larger than the one of metabolism. While we think that this is a true finding, some physical issues should be discussed. LSI detects laser light at $785 \mathrm{~nm}$ where absorption is weak. This leads to multiple scattering of light, and thus structures in certain depth (say $0.5 \mathrm{~mm}$ ) might appear somewhat blurred. Imaging of flavoprotein fluorescence on the other hand uses visible light around $500 \mathrm{~nm}$ where absorption is strong and multiple scattering is suppressed because of absorption. The emitted light thus leaves the tissue in a rather direct way (without being scattered many times). Although this topic needs to be addressed thoroughly for both methods with modelling and experimental approaches, it is unlikely that this blurring effect can explain the large difference in the extent of the activated area (Fig. 3). The different wavelengths of the two methods also have a direct effect on the cortical depth that is being probed. Also this issue requires further research. Durduran et al. (2004) report that they expect most of the signal from $785 \mathrm{~nm}$ laser speckle to originate from a cortical depth of about $0.3 \mathrm{~mm}$. Surprisingly, this would be very similar to the depth of flavoprotein autofluorescence changes reported Shibuki et al. (2003).

In this work we applied pixelwise modelling. This is a powerful tool to extract valuable information from time-activity curves. The main advantage is that it allows the calculation of user-independent parametric maps that are based on each pixel's temporal characteristics. This is not the case in the often-used subtraction methods, where, for example, the mean maximum or minimum response frame is used. The employed gamma curve is appropriate for both methods because it adequately fits the data, as can be seen in Fig. 2. This figure also demonstrates the excellent signal to noise ratio of both methods, which allows functional mapping on the single trial level without averaging, reducing the length of experiments.

A disadvantage of both methods is that they are not quantitative (i.e. they do not yield measure in absolute units, e.g. $\mathrm{ml} / \mathrm{min} / \mathrm{g}$ or $\mu \mathrm{mol} / \mathrm{min} / \mathrm{g}$ ) as they are implemented in the present study. However, for many studies in basic neuroimaging research quantitative information is needed. Theoretically, LSI can be used quantitatively (Dunn et al., 2001); however, this is subject to further development and will have to be validated with established quantitative methods, e.g. beta probe studies (Weber et al., 2003).

\section{Conclusions}

Using concurrent laser speckle and flavoprotein AF imaging the present study shows that increases in cerebral oxidative metabolism in response to sensory stimulation is considerably faster and more localized than the CBF response. It is mainly the latter result that has important implications for the interpretation and development of noninvasive neuroimaging methods, such as fMRI. It confirms that the development of methods probing the metabolic response would be a promising way to increase the spatial resolution of functional imaging.

\section{Acknowledgements}

We thank K. Mikolajczyk for the help with the implementation of the pixelwise modelling tools in PMOD and Schäfter und Kirchhoff, Hamburg, Germany and Leica Microsystems, Glattbrugg, Switzerland for the efficient collaboration. We are greatful to Katsuei Shibuki and Christopher Moore for important discussions and help. This study was supported in part by the Swiss National Science Foundation grant 205321-104282/1.

\section{Abbreviations}

$\mathrm{AF}$, autofluorescence; ANOVA, analysis of variance; $\mathrm{CBF}$, cerebral blood flow; $\mathrm{CBV}$, cerebral blood volume; fMRI, functional magnetic resonance imaging; LSI, laser speckle imaging; PET, positron emission tomography; SNR, signalto-noise ratio.

\section{References}

Ances, B.M., Greenberg, J.H. \& Detre, J.A. (1999) Laser doppler imaging of activation-flow coupling in the rat somatosensory cortex. Neuroimage, $\mathbf{1 0}$, 716-723.

Armstrong-James, M. \& Callahan, C.A. (1991) Thalamo-cortical processing of vibrissal information in the rat. II. spatiotemporal convergence in the thalamic ventroposterior medial nucleus (VPm) and its relevance to generation of receptive fields of S1 cortical 'barrel' neurones. J. Comp. Neurol., 303, 211-224.

Barlow, C.H., Harden, W.R., 3rd, Harken, A.H., Simson, M.B., Haselgrove, J.C., Chance, B., O’Connor, M. \& Austin, G. (1979) Fluorescence mapping of mitochrondrial redox changes in heart and brain. Crit. Care Med., 7, 402406.

Berne, B.J. \& Pecora, R. (1976) Dynamic Light Scattering. John Wiley and Sons, New York.

Bonhoeffer, T. \& Grinvald, A. (1996) Optical imaging based on intrinsic signals. The methodology. In Toga, A.W. \& Mazziotta, J.C. (eds), Brain Mapping. The Methods. Academic Press, London, pp. 55-97.

Bonvento, G., Charbonne, R., Correze, J.L., Borredon, J., Seylaz, J. \& Lacombe, P. (1994) Is alpha-chloralose plus halothane induction a suitable anesthetic regimen for cerebrovascular research? Brain Res., 665, 213 221.

Briers, J.D. (2001) Laser Doppler, speckle and related techniques for blood perfusion mapping and imaging. Physiol. Meas., 22, R35-R66.

Davis, T.L., Kwong, K.K., Weisskoff, R.M. \& Rosen, B.R. (1998) Calibrated functional MRI: mapping the dynamics of oxidative metabolism. Proc. Natl. Acad. Sci. USA, 95, 1834-1839.

Dunn, A.K., Bolay, H., Moskowitz, M.A. \& Boas, D.A. (2001) Dynamic imaging of cerebral blood flow using laser speckle. J. Cereb. Blood Flow Metab., 21, 195-201.

Dunn, A.K., Devor, A., Bolay, H., Andermann, M.L., Moskowitz, M.A., Dale, A.M. \& Boas, D.A. (2003) Simultaneous imaging of total cerebral hemoglobin concentration, oxygenation, and blood flow during functional activation. Opt. Lett., 28, 28-30. 
Duong, T.Q., Kim, D.S., Ugurbil, K. \& Kim, S.G. (2001) Localized cerebral blood flow response at submillimeter columnar resolution. Proc. Natl. Acad. Sci. USA, 98, 10904-10909.

Durduran, T., Burnett, M.G.YuG., Zhou, C., Furuya, D., Yodh, A.G., Detre, J.A. \& Greenberg, J.H. (2004) Spatiotemporal quantification of cerebral blood flow during functional activation in rat somatosensory cortex using laser-speckle flowmetry. J. Cereb. Blood Flow Metab., 24, 518-525.

Frostig, R.D., Lieke, E.E., Ts'o, D.Y. \& Grinvald, A. (1990) Cortical functional architecture and local coupling between neuronal activity and the microcirculation revealed by in vivo high-resolution optical imaging of intrinsic signals. Proc. Natl. Acad. Sci. USA, 87, 6082-6086.

Gerrits, R.J., Stein, E.A. \& Greene, A.S. (1998) Blood flow increases linearly in rat somatosensory cortex with increased whisker movement frequency. Brain Res., 783, 151-157.

Grinvald, A., Lieke, E., Frostig, R.D., Gilbert, C.D. \& Wiesel, T.N. (1986) Functional architecture of cortex revealed by optical imaging of intrinsic signals. Nature, 324, 361-364.

Haselgrove, J.C., Bashford, C.L., Barlow, C.H., Quistorff, B., Chance, B. \& Mayevsky, A. (1990) Time resolved 3-dimensional recording of redox ratio during spreading depression in gerbil brain. Brain Res., 506, 109-114.

Jones, M., Berwick, J. \& Mayhew, J. (2002) Changes in blood flow, oxygenation, and Volume following extended stimulation of rodent barrel cortex. Neuroimage, 15, 474-487.

Lauritzen, M. \& Fabricius, M. (1995) Real time laser-Doppler perfusion imaging of cortical spreading depression in rat neocortex. Neuroreport, 6 , 1271-1273.

Lewis, D.V. \& Schuette, W.H. (1976) NADH fluorescence, $[\mathrm{K}+] 0$ and oxygen consumption in cat cerebral cortex during direct cortical stimulation. Brain Res., 110, 523-535.

Logothetis, N.K. \& Wandell, B.A. (2004) Interpreting the BOLD signal. Annu. Rev. Physiol., 66, 735-769.

Lothman, E., Lamanna, J., Cordingley, G., Rosenthal, M. \& Somjen, G. (1975) Responses of electrical potential, potassium levels, and oxidative metabolic activity of the cerebral neocortex of cats. Brain Res., 88, 15-36.

Malonek, D. \& Grinvald, A. (1996) Interactions between electrical activity and cortical microcirculation revealed by imaging spectroscopy: implications for functional brain mapping. Science, 272, 551-554.

Martindale, J., Mayhew, J., Berwick, J., Jones, M., Martin, C., Johnston, D., Redgrave, P. \& Zheng, Y. (2003) The hemodynamic impulse response to a single neural event. J. Cereb. Blood Flow Metab., 23, 546-555.

Mayevsky, A. \& Chance, B. (1975) Metabolic responses of the awake cerebral cortex to anoxia hypoxia spreading depression and epileptiform activity. Brain Res., 98, 149-165.
Mayevsky, A. \& Chance, B. (1983) Multisite measurements of NADH redox state from cerebral cortex of the awake animal. Adv. Exp. Med. Biol., 159, $143-155$.

Moore, C.I. \& Nelson, S.B. (1998) Spatio-temporal subthreshold receptive fields in the vibrissa representation of rat primary somatosensory cortex. J. Neurophysiol., 80, 2882-2892.

Murakami, H., Kamatani, D., Hishida, R., Takao, T., Kudoh, M., Kawaguchi, T., Tanaka, R. \& Shibuki, K. (2004) Short-term plasticity visualized with flavoprotein autofluorescence in the somatosensory cortex of anaesthetized rats. Eur. J. Neurosci., 19, 1352-1360.

Ohno, K., Pettigrew, K.D. \& Rapoport, S.I. (1979) Local cerebral blood flow in the conscious rat as measured with 14C-antipyrine, 14C-iodoantipyrine and 3H-nicotine. Stroke, 10, 62-67.

Reinert, K.C., Dunbar, R.I.R., Gao, W., Chen, G. \& Ebner, T.J. (2004) Flavoprotein autofluorescence imaging of neuronal activation in the cerebellar cortex in vivo. J. Neurophysiol., 92, 199-211.

Sakurada, O., Kennedy, C., Jehle, J., Brown, J.D., Carbin, G.L. \& Sokoloff, L. (1978) Measurement of local cerebral blood flow with iodo [14C] antipyrine. Am. J. Physiol., 234, H59-H66.

Sheth, S.A., Nemoto, M., Guiou, M., Walker, M., Pouratian, N., Hageman, N. \& Toga, A.W. (2004b) Columnar specificity of microvascular oxygenation and Volume responses: implications for functional brain mapping. J. Neurosci., 24, 634-641.

Sheth, S.A., Nemoto, M., Guiou, M., Walker, M., Pouratian, N. \& Toga, A.W. (2004a) Linear and nonlinear relationships between neuronal activity, oxygen metabolism, and hemodynamic responses. Neuron, 42, 347-355.

Shibuki, K., Hishida, R., Murakami, H., Kudoh, M., Kawaguchi, T., Watanabe, M., Watanabe, S., Kouuchi, T. \& Tanaka, R. (2003) Dynamic imaging of somatosensory cortical activity in the rat visualized by flavoprotein autofluorescence. J. Physiol. (Lond.), 549, 919-927.

Thompson, J.K., Peterson, M.R. \& Freeman, R.D. (2003) Single-neuron activity and tissue oxygenation in the cerebral cortex. Science, 299, 1070-1072.

Vanzetta, I., Slovin, H., Omer, D.B. \& Grinvald, A. (2004) Columnar resolution of blood Volume and oximetry functional maps in the behaving monkey; implications for FMRI. Neuron, 42, 843-854.

Weber, B., Spath, N., Wyss, M., Wild, D., Burger, C., Stanley, R. \& Buck, A. (2003) Quantitative cerebral blood flow measurements in the rat using a betaprobe and H2, 15O. J. Cereb. Blood Flow Metab., 23, 1455-1460.

Welker, C. (1971) Microelectrode delineation of fine grain somatotopic organization of (SmI) cerebral neocortex in albino rat. Brain Res., 26, 259-275.

Zhu, J.J. \& Connors, B.W. (1999) Intrinsic firing patterns and whisker-evoked synaptic responses of neurons in the rat barrel cortex. J. Neurophysiol., $\mathbf{8 1}$, $1171-1183$. 